\title{
Status and Plans for the CERN Accelerator Complex
}

\author{
Paul Collier ${ }^{1}$ \\ CERN \\ 1 Esplanade des Particules, Geneva 23, Switzerland \\ E-mail: Paul.Collierecern.ch
}

CERN hosts the largest accelerator complex in the world. The flagship machine is the LHC providing proton and heavy ion collisions at the energy frontier to 4 main experiments and a number of smaller ones. In addition, the LHC injector complex provides beams at a variety of energies and particle types to several experimental areas, serving many fixed-target experiments and together forming the CERN physics diversity programme. After a short recap of the history of LHC run 2, the status of the presently approved programme will be outlined. This includes the major upgrades to both the LHC and the injector complex as well as a number of upgraded or new facilities coming online after the present long shutdown.

The European Particle Physics Strategy Update (EPPSU) is presently in progress to determine the priorities for the field in Europe during the coming years. As part of the input to this process, CERN has provided details of a number of projects that could be realized at CERN. These include studies for the next energy frontier machine as well possiblenew facilities to enhance the diversity physics programme. The major studies for CLIC and FCC will be presented as well as a few of the other projects where significant additional infrastructure or enhancements to the complex are needed. It is hoped that the EPPSU will set the direction for taking some of the proposals forward within the constraints of the available resources of the organization. 


\section{Introduction}

The CERN complex is presently shut down for major maintenance and upgrades. This long Shutdown (LS2) follows a very successful run 2 of the LHC (2015-1018) and will be used to complete a major upgrade of the LHC Injectors in preparation for HL-LHC as well as preparatory works for the HL-LHC upgrade itself. In 2021 the complex will be restarted for LHC run 3 and then run for 3 years before the next long shutdown, where the main HL-LHC changes will be implemented. From then on, the LHC will run with a significantly enhanced collision rate to deliver $\sim 250 \mathrm{fb}^{-1} /$ year to the two general purpose experiments, ATLAS and CMS.

In parallel to LHC, CERN hosts a wide variety of other experiments clustered around a number of experimental facilities. These facilities are attached to the different machines forming the LHC injector complex and operate at a wide range of energies. These different facilities are known as the diversity physics programme at CERN. During LS2, major changes and upgrades are also planned in some of these facilities as part of the approved CERN programme. During Run 3 many of these will be fully exploited for the first time, giving exciting prospects for the physics programme.

Beyond the presently approved programme, studies have been undertaken for possible future facilities at CERN. These have all been submitted to the European Particle Physics Strategy Update (EPPSU) which is presently in progress and due to report to CERN council in mid-2020. As part of the input to this process, CERN has provided details of a number of projects that could be realized at CERN. These include studies for the next energy frontier machine as well as a number of possible facilities to enhance the diversity physics programme. It is hoped that the EPPSU will set the direction for taking some of the proposals forward within the constraints of the available resources of the organization.

\section{A Short History of LHC Run 2}

LHC run 2 began in mid-2015 and ended in December 2018 with a total of $160 \mathrm{fb}^{-1}$ of protonproton collisions at $13 \mathrm{TeV}$ delivered to ATLAS and CMS and around $6.7 \mathrm{fb}^{-1}$ to $\mathrm{LHCb}$. In this mode, ALICE also took low pile-up p-p collisions for a total integrated luminosity of $33 \mathrm{pb}^{-1}$.

As well as proton-proton collisions, LHC provided runs with $\mathrm{Pb}-\mathrm{Pb}$ ions as well as $\mathrm{p}-\mathrm{Pb}$. In addition, there was a short Xe-Xe run, as well as a host of "special runs". For lack of space only the $\mathrm{p}$-p high luminosity runs will be described here.

During run 2 several important milestones were reached in the LHC:

- The machine demonstrated reliable and high-efficiency operation with $6.5 \mathrm{TeV}$ beams having a very high stored beam energy on the machine ( $>300 \mathrm{MJ} /$ beam).

- The 20ns bunch spacing was fully deployed and validated for high luminosity operation allowing the machine to operate with $>2500$ bunches.

- The LHC design luminosity of $1 \times 10^{+34} \mathrm{~cm}^{-2} \mathrm{~s}^{-1}$ was quickly reached and subsequently exceeded by a factor of 2 .

- Several concepts needed for operation during the HL-LHC era were tested and subsequently deployed.

The integrated luminosity per year in p-p operation is shown in Fig. 1. 


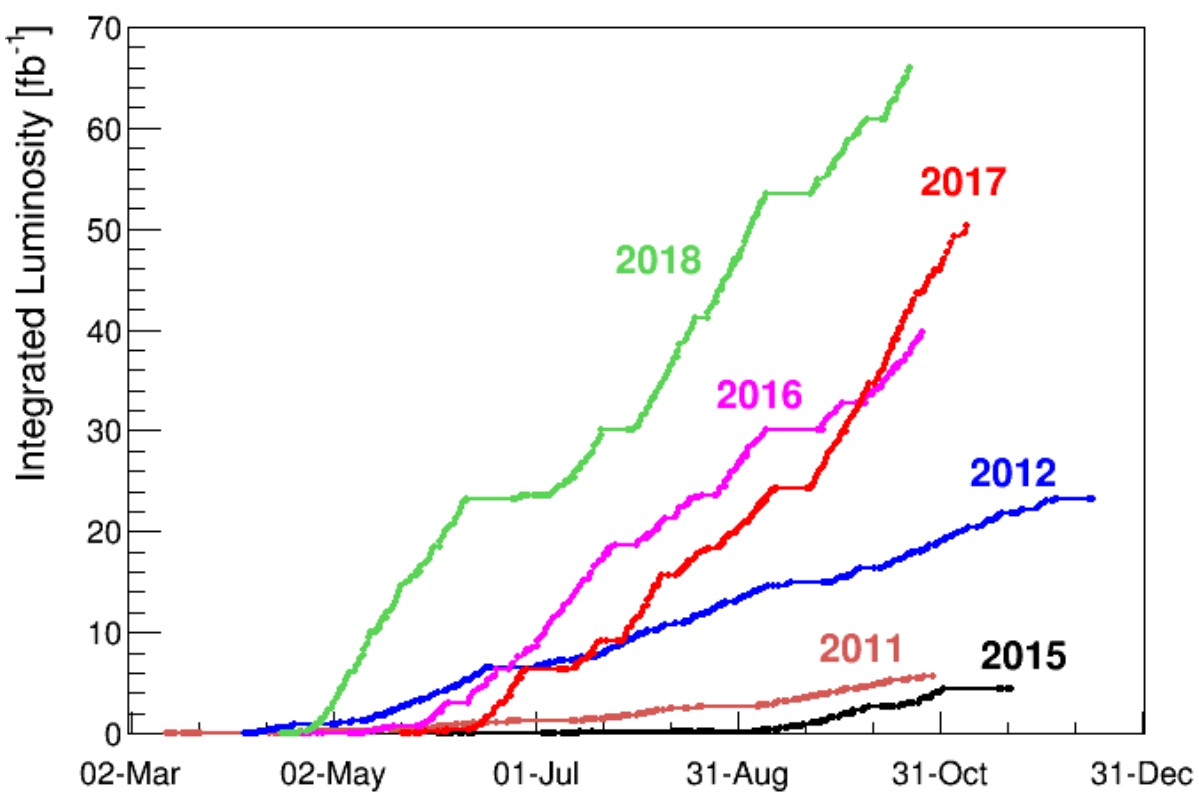

Figure 1: LHC p-p Integrated Luminosity per year giving a total for the LHC to date of 189fb-1 and 160fb-1 over Run 2 (2015-2018)

The LHC machine was beautifully designed and built extremely well. In addition, the beam dynamics and control are now well mastered and understood. These generated "margins" that were exploited to increase the luminosity. The main advantage taken was to progressively reduce the $\beta^{*}$ at the ATLAS and CMS interaction points. The design value was $55 \mathrm{~cm}$, but tight control over the orbit and beta-beating allowed this to reach $30 \mathrm{~cm}$ at the beginning of a physics fill and be further reduced to $25 \mathrm{~cm}$ towards the end. In addition, new concepts for preparing the beam in the injectors were deployed allowing a significant increase in beam brightness in the LHC. Towards the end of run 2, it was necessary to level the luminosity at the beginning of a physics fill to $2 \times 10^{+34} \mathrm{~cm}^{-2} \mathrm{~s}^{-1}$ in order to limit the event pile-up in the experiments. Later in the fill, antilevelling techniques were used to increase the luminosity as the beam intensity decayed. This included a mechanism for continuously reducing the crossing angle as a function of bunch intensity and later a $\beta^{*}$ reduction in steps from 30 to $25 \mathrm{~cm}$. Although these anti-levelling mechanisms only contributed a few percent to the total integrated luminosity, they were important proof-of concept deployments for HL-LHC, where they form a major part of the strategy for luminosity levelling and control.

A storage collider, such as LHC, can never be $100 \%$ efficient as time is needed to inject accelerate and prepare the beams for stable beam operation as well as recovery and a ramp down to injection energy after a fill was dumped. However, the LHC managed to maintain an excellent level of $\sim 50 \%$ of the total scheduled physics time actually in stable beam operation. The remaining time was split fairly evenly between the time needed to for the operational cycle and down time due to faults. 


\section{The Approved Programme: Prospects for Run3 and Beyond}

In this section the various approved projects which form the present medium-term plan for the organization are outlined along with their present status and plans. This covers the LHC injector upgrade, the HL-LHC project and a number of smaller projects aimed at enhancing the other scientific programmes at CERN.

\subsection{The LHC Injector Upgrade Project}

The LHC Injector Upgrade Project (LIU) [1] aims to deliver the beams required to meet the HL-LHC goal of $3 \mathrm{ab}^{-1}$ integrated luminosity in $\sim 12$ years of operation. To achieve this target the intensity of the injected beam has to be doubled and its brightness multiplied by a factor 2.5 $\left(2.3 \times 10^{+11}\right.$ protons/bunch with $25 \mathrm{~ns}$ bunch spacing with an emittance of $\left.2.1 \mu \mathrm{m}\right)$. A similar challenge is set for lead $(\mathrm{Pb})$ ions. Meeting these goals requires upgrades to all parts of the injector complex, from the source to the final elements of the SPS before injection into the LHC.

The main changes are:

- The construction of a new $160 \mathrm{MeV}, \mathrm{H}^{-}$linac (Linac4) to replace the 40 -year-old Linac2 which delivered $\mathrm{p}^{+}$at $50 \mathrm{MeV}$,

- Increase the injection energy of the PS booster and upgrade of the injection systems for $\mathrm{H}^{-}$injection,

- Increase of the extraction energy of the booster from the present $1.4 \mathrm{GeV}$ to $2 \mathrm{GeV}$,

- Upgrade of the PS transfer and injection to $2 \mathrm{GeV}$

In addition, there will be major upgrades to many systems in the injector chain to cope with the increased bunch intensity and beam brightness.

The installation of all the LIU equipment is taking place during the present long shutdown (LS2) and will be completed in 2020. The machines of the injector complex will be progressively re-commissioned during the second half of the year to be ready for LHC early in 2021. Initially the injectors will be commissioned to deliver an intensity equivalent to that achieved during run 2 and the intensity will be progressively ramped up during run 3 where the luminosity in the LHC will be limited to $2 \times 10^{+34}$ (a pile-up of 60 ). An integrated luminosity of around $160 \mathrm{fb}^{-1}$ should be achievable during run 3 .

\subsection{The HL-LHC project}

As already mentioned, the aim of the HL-LHC project [2] is to deliver $3 \mathrm{ab}^{-1}$ to ATLAS and CMS at a rate of $\sim 250 \mathrm{fb}^{-1}$ per year with a levelled luminosity of $5 \times 10^{+34} \mathrm{~cm}^{-2} \mathrm{~s}^{-1}$ in order to limit the pile-up in the experiments to 140 events per crossing. In order to achieve this, many parts of the accelerator will be upgraded, including:

- New wide-aperture final focus quadrupoles based on $\mathrm{Nb}_{3} \mathrm{Sn}$ together with many other new NbTi magnets in the interaction region giving access to stronger focusing at the interaction point,

- A collimation system upgrade to provide enhanced beam cleaning and including new, 11Tesla $\mathrm{Nb}_{3} \mathrm{Sn}$ dipoles to allow collimators to be installed in 2 of the arcs,

- The installation of crab cavities at either side of ATLAS and CMS to offset the crossing angle reduction factor,

- A cryogenics upgrade including 2 new cryogenic plants. 
- New cold powering systems and machine protection enhancements,

- Upgrades to beam instrumentation.

In order to install all the new equipment significant new infrastructure is needed at point 1 (ATLAS) and point 5 (CMS) of the LHC. This includes underground caverns and galleries as well as several surface buildings. The civil engineering work to prepare this infrastructure is underway during LS2. The main installations for HL-LHC will take place during a 30-month shutdown starting in 2024.

\subsection{Approved Projects of the Diversity Physics Programme at CERN}

Several projects are either underway, or will be completed during LS2 that will enhance the physics potential of many non-LHC experiments at CERN. These include:

- HIE-Isolde [3]. Isolde is a radioactive ion beam (RIB) facility at the PS booster giving access to a wide range of isotopes for atomic, nuclear and solid-state physics. HIEIsolde is a new post-accelerator to provide RIB at energies up to $10 \mathrm{MeV} / \mathrm{u}$. Based on superconducting $1 / 4$-wave cavities, the facility has been progressively installed and will run for the first time at its design energy after LS2

- ELENA [4]. The Antiproton Decellerator (AD) facility provides low-energy antiprotons to a number of experiments most of which create and study anti-hydrogen atoms. The ELENA project was initiated in 2013 to provide even lower energies for the anti-protons and hence increase the yield of anti-hydrogen by a factor 10-100. ELENA is a post decelerator synchrotron taking the beam from the $\mathrm{AD}$ at $5 \mathrm{MeV}$ and decelerating it to $100 \mathrm{keV}$ before delivering the cooled beam to several experiments simultaneously. The ELENA ring was completed and commissioned during 2018 and will be connected to the existing experiments via new electrostatic beam-lines during LS2.

- The East Hall is a fixed target facility attached to the PS, delivering beam to a number of experiments and an irradiation facility. The facility was built almost 60 years ago. During LS2 a major renovation project is underway to modernize and enhance the beam facilities and infrastructure. In addition, rebuilding the beam-lines with pulsed elements will allow a significant saving in energy

- The North Area is a huge fixed-target facility at the SPS serving a large number of experiments as well as providing test beams for detector development. It consists of 4 main targets and several $\mathrm{km}$ of primary and secondary beam-lines. The facility dates from the 1970's and needs refurbishment. Some upgrades have already been implemented, however a major refurbishment of the whole facility will start during LS3 (2025) and continue for several years.

- The Neutrino Platform is a new facility attached to the SPS North Area. It is designed to support and foster the European participation in neutrino experiments around the world and consists of 2 major experimental regions presently housing single and dual phase liquid argon detector prototypes for DUNE. Both regions are served with charged particle beam lines from the North Area. The single-phase detector has already been commissioned and took beam during 2018. The dual-phase prototype is being commissioned now and will take beam after LS2

- AWAKE [5] is a proof-of-principle facility to study proton driven plasma wakefield acceleration. During 2018 it demonstrated the necessary seeded self-modulation in the 
proton beam and successfully accelerated a witness electron beam with a peak accelerating gradient in the plasma of $200 \mathrm{MV} / \mathrm{m}$. A programme has now been approved to continue the studies including an upgrade to allow it to accelerate the beam further while preserving the electron beam quality. This programme could become the precursor of a user facility in the future.

\section{The European Particle Physics Strategy Update and Directions for the Future}

The European Particle Physics Strategy Update process (EPPSU) is presently underway and will produce a strategy update for approval by CERN council in May 2020. In preparation for this, inputs from the community were solicited and discussed in an open symposium earlier this year. Here I shall only discuss inputs that directly concern CERN future experiments and facilities and these broadly break down into two types: future energy frontier machines and enhancements to the non-collider physics programme.

\subsection{Energy Frontier Studies at CERN}

Consensus seems to be emerging that the next energy frontier machine should be a "Higgsfactory" allowing a detailed study of its properties. It is generally accepted that such a machine should be an $\mathrm{e}^{+} \mathrm{e}^{-}$collider. CERN has undertaken two major studies on possible energy frontier machines. Both offer good potential for studying the Higgs, but have different capabilities concerning additional physics potential.

\subsubsection{CLIC}

CLIC [6] is a linear $\mathrm{e}^{+} \mathrm{e}^{-}$collider to be constructed in phases with the first stage (380GeV $\mathrm{CoM}$ ) giving access to Higgs and top physics. Later extensions to the machine could give collision energies up to $3 \mathrm{TeV}$, which would give indirect sensitivity to energy scales up to $\Lambda \sim 100 \mathrm{TeV}$ [7]. In preparation for the EPPSU a project implementation plan has been published [8] detailing both the costs and a possible schedule for the facility. The main parameters at the 3 energy stages are given in table 1.

Table 1: Relevant CLIC Parameters

\begin{tabular}{|l|c|c|c|c|}
\hline \multicolumn{1}{|c|}{ Parameter } & Unit & Stage 1 & Stage 2 & Stage 3 \\
\hline Collision Energy & $\mathrm{GeV}$ & 380 & 1500 & 3000 \\
\hline Tunnel Length & $\mathrm{Km}$ & 11 & 29 & 50 \\
\hline Accelerating Gradient & $\mathrm{MV} / \mathrm{m}$ & 72 & $72 / 100$ & $72 / 100$ \\
\hline Pulse Length & $\mathrm{ns}$ & 244 & 244 & 244 \\
\hline Luminosity & $10^{+34} \mathrm{~cm}^{-2} \mathrm{~s}^{-1}$ & 1.5 & 3.7 & 5.9 \\
\hline Repetition Rate & $\mathrm{Hz}$ & 50 & 50 & 50 \\
\hline Bunches per Train & & 352 & 312 & 312 \\
\hline Particles per Bunch & $10^{9}$ & 5.2 & 3.7 & 3.7 \\
\hline Beam Size at the IP $\left(\sigma_{\mathrm{y}} / \sigma_{\mathrm{x}}\right)$ & $\mathrm{nm}$ & $2.9 / 149$ & $1.5 / 60$ & $1 / 40$ \\
\hline Annual Energy Consumption & $\mathrm{TWh}$ & 0.8 & 1.7 & 2.8 \\
\hline Construction Cost & $\mathrm{BCHF}$ & 5.9 & +5.1 & +7.3 \\
\hline
\end{tabular}


The outline schedule for CLIC shows that after a 6-year preparatory phase and a 7-year construction and commissioning period physics at $380 \mathrm{GeV}$ could begin. Being a linear collider there would be a single interaction point hosting either one major experiment, or 2 in a push-pull configuration. Depending on the scenarios for the later energy upgrades, the facility would operate for around 30 years.

\subsubsection{FCC}

The Future Circular Collider studies (FCC) cover a number of possible machines sharing the same $100 \mathrm{~km}$ infrastructure [9]. The present plan would be an initial $\mathrm{e}^{+} \mathrm{e}^{-}$collider with collision energies in the range $90 \mathrm{GeV}-365 \mathrm{GeV}[10]$. This would open the possibility for a re-exploration of the electro-weak regime with collisions at the Z-peak and WW-threshold as well as for Higgs $(240 \mathrm{GeV})$ and Top $(365 \mathrm{GeV})$ production. Presently the design is for 2 experiments, however this could be increased to 4 . To cover the whole programme, the $\mathrm{e}^{+} \mathrm{e}^{-}$collider would operate for physics for at least 15 years. Following this the basic infrastructure would be re-used to house a hadron-hadron collider with a centre of mass energy for pp collisions of $100 \mathrm{TeV}$ [11] and the possibility for heavy ion collisions. In concurrent operation, opportunities would also exist for proton-electron and electron-lead collisions. The summary of the performance for each stage of the $\mathrm{FCC}$ is given in table 2.

Table 2: Performance Estimates for the various FCC options

\begin{tabular}{|c|c|c|c|c|}
\hline & $\sqrt{s}$ & $\mathrm{~L} / \mathrm{IP}\left(\mathrm{cm}^{-2} \mathrm{~s}^{-1}\right)$ & Int. $L / I P\left(a b^{-1}\right)$ & Comments \\
\hline $\begin{array}{l}\mathrm{e}^{+} e^{-} \\
\text {FCC-ee }\end{array}$ & $\begin{array}{cr}\sim 90 \mathrm{GeV} & \mathrm{Z} \\
160 & \mathrm{wW} \\
240 & \mathrm{H} \\
\sim 365 & \text { top }\end{array}$ & $\begin{array}{l}230 \times 10^{34} \\
28 \\
8.5 \\
1.5\end{array}$ & $\begin{array}{l}75 \mathrm{ab}^{-1} \\
5 \\
2.5 \\
0.8\end{array}$ & $\begin{array}{l}2 \text { experiments } \\
\text { Total } \sim 15 \text { years of } \\
\text { operation }\end{array}$ \\
\hline $\begin{array}{l}\text { pp } \\
\text { FCC-hh }\end{array}$ & $100 \mathrm{TeV}$ & $\begin{array}{l}5 \times 10^{34} \\
30\end{array}$ & $\begin{array}{l}2.5 a b^{-1} \\
15\end{array}$ & $\begin{array}{l}2+2 \text { experiments } \\
\text { Total } \sim 25 \text { years of } \\
\text { operation }\end{array}$ \\
\hline $\begin{array}{l}\mathrm{PbPb} \\
\text { FCC-hh }\end{array}$ & ${\sqrt{S_{\mathrm{NN}}}}=39 \mathrm{TeV}$ & $3 \times 10^{29}$ & $100 \mathrm{nb}^{-1 / \text { run }}$ & $\begin{array}{l}1 \text { run = } 1 \text { month } \\
\text { operation }\end{array}$ \\
\hline $\begin{array}{l}\text { ep } \\
\text { Fcc-eh }\end{array}$ & $3.5 \mathrm{TeV}$ & $1.510^{34}$ & $2 a b^{-1}$ & $\begin{array}{l}60 \mathrm{GeV} \text { e- from } \mathrm{ERL} \\
\text { Concurrent operation } \\
\text { with pp for } \sim 20 \text { years }\end{array}$ \\
\hline $\begin{array}{l}\text { e-Pb } \\
\text { Fcc-eh }\end{array}$ & $\sqrt{ } \mathrm{s}_{\mathrm{eN}}=2.2 \mathrm{TeV}$ & $0.510^{34}$ & $1 \mathrm{fb}^{-1}$ & $\begin{array}{l}60 \mathrm{GeV} \text { e- from ERL } \\
\text { Concurrent operation } \\
\text { with } \mathrm{PbPb}\end{array}$ \\
\hline
\end{tabular}

As part of the study, a cost estimate has been made for the cost and energy consumption of the 2 major machines (FCC-ee and FCC-hh) and is given in Table 3.

Table 3: Cost and Power Consumption Estimates for FCC

\begin{tabular}{|c|c|c|c|}
\multicolumn{2}{c}{$\begin{array}{c}\text { Cost-estimate } \\
\text { /BCHF }\end{array}$} & $\begin{array}{c}\text { AC-Power } \\
\text { /MW }\end{array}$ & Comments \\
\hline Infrastructure & 5.5 & & $100 \mathrm{~km}$ tunnel and surface infrastructure \\
\hline FCC-ee & 5 & $260-350$ & +1.1 BCHF for the Top stage $(365 \mathrm{GeV})$ \\
\hline FCC-hh & 17 & 580 & \\
\hline
\end{tabular}




\subsubsection{Non-Collider Physics Options}

In 2016 a study (Physics Beyond Colliders, PBC) was launched at CERN to provide input to the EPPSU concerning the development and future potential non-collider facilities based around the present CERN infrastructure [12]. From this study number of experiments and facilities are proposed for implementation. These range from upgrades to existing fixed-target experiments to major additions to the experimental facilities at CERN and covering a range of physics opportunities in QCD, rare decays, hidden sector physics, long-lived particles and even non-accelerator based experiments. The cost and timelines for these proposals covers a wide range from a few MCHF and almost construction ready to over a hundred MCHF and significant development and construction time required. Figure 2 summarizes the list of proposals submitted to the EPPSU. All proposals cannot be covered here and are described in [12] and associated documents. Instead, mention here will be made of only two that require either major additional facilities, or a significant change to the operation of the present machines.

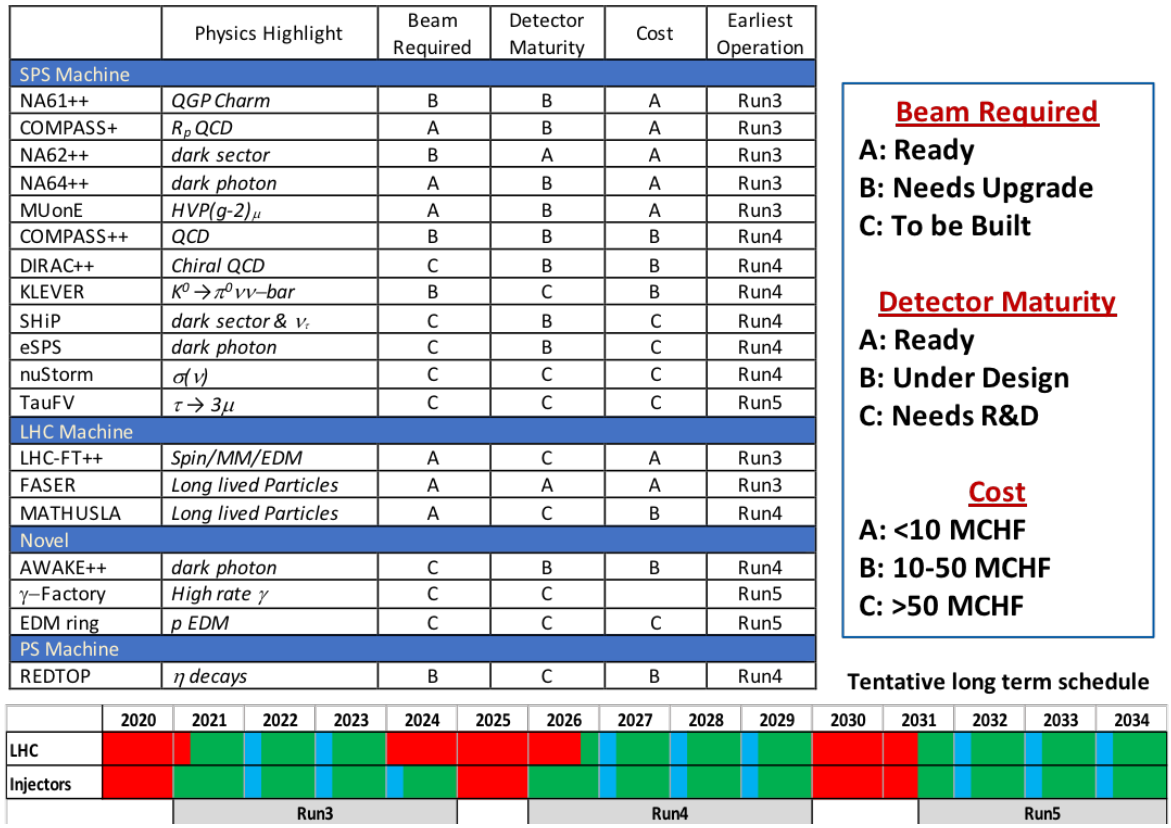

Figure 2: PBC Study, Experiment Readiness and Cost

Exploring the hidden sector with a "beam-dump" facility was a common theme for several submissions. As well as operating existing (upgraded) experiments in beam-dump mode, a proposal for a dedicated beam-dump facility for the SPS North area is included [13]. Although initially serving the proposed experiments SHiP and TauFV, it would become a general experimental facility. A complimentary facility to study light dark matter has also been proposed. This is based on accelerating electrons in the SPS to $16 \mathrm{GeV}$ and extracting them through existing transfer lines to a new experimental hall hosting the LDMX experiment [14]. The electrons would be produced in a $3.5 \mathrm{GeV}$ linac built in the SPS west area using CLIC, X-band structures and which could serve other experiments based on a beam dump as well as being a useful facility for general accelerator R\&D. Both the facilities outlined above require significant resources as they need significant new infrastructure and it is not clear that both can go ahead, with the bottle-neck being the use of the SPS. 


\section{Conclusions}

CERN is presently executing a $\sim 1.4 \mathrm{BCHF}$ project to upgrade the LHC, its injectors and the experiments. This aims to deliver $3 \mathrm{ab}^{-1}$ to the CMS and ATLAS as well as delivering increased luminosity to LHCb and ALICE. Construction is in full swing and the peak of the spending occurs in the coming few years. As a result, resources are limited for any new initiatives recommended by the 2020 EPPSU until around 2025 .

For the future, CERN has prepared two detailed studies for a future energy-frontier machine. Both would initially be e+e- "Higgs-factories", followed by either a hadron collider (100TeV), or a lepton collider $(3 \mathrm{TeV})$. Both energy frontier projects require an initial preparatory phase of 6-8 years. If efforts are concentrated on one of the projects, only relatively modest additional resources would be required during the next 5 years. CERN is therefore hoping for a clear recommendation from the EPPSU on which route to take. Whatever path is chosen, the next 5 years will have to be spent preparing a full TDR as well as a detailed site design and discussions with the host state authorities. Last, but not least, the chosen machine would have to be established as a world-wide facility with a corresponding funding model to secure the necessary resources.

In preparation for the EPPSU, CERN set up the Physics Beyond Colliders study group in order to solicit suggestions for new non-collider facilities and experiments that could make use of the present infrastructure. A large number of proposals have been made covering a wide range of physics opportunities which are complimentary to the collider-based physics programme. All proposals have been submitted as input to the EPPSU and once its priorities and recommendations are known, some of these proposals could move forward towards a full TDR and, depending on the availability of resources, eventual construction.

\section{References}

[1] H Damerau et.al. LHC Injectors Upgrade, Technical Design Report, Vol.1: Protons, CERN-ACC2014-0337, CERN, Geneva, 2014.

[2] G Apollinari et. al. (Ed.), High-Luminosity Large Hadron Collider (HL-LHC): Technical Design Report, CERN-2017-007-M, CERN, Geneva, 2017.

[3] Y Kadi (Ed.), M A Fraser (Ed.) \& A Papageorgiou-Koufidou (Ed.), HIE-ISOLDE: Technical design report for the energy upgrade, CERN-2018-002-M, CERN, Geneva, 2018

[4] V Chohan et. al., ELENA Technical Design Report, CERN EDMS, https:/ledms.cern.ch/document/1303646/1.0

[5] A Caldwell, E Gschwendtner, K Lotov, P Muggli \& M Wing, AWAKE Design Report: A ProtonDriven Plasma Wakefield Experiment at CERN, CERN-SPSC-2013-013, CERN, Geneva, 2013.

[6] M. Aicheler et.al. (Ed.), A Multi-TeV Linear Collider Based on CLIC Technology, CLIC Conceptual Design Report, CERN-2012-007, CERN, Geneva, 2012.

[7] P Lebrun et. al. (Ed.), The CLIC Programme: Towards a Staged $e^{+} e^{-}$Linear Collider Exploring the Terascale, CERN-2012-005, CERN, Geneva, 2012.

[8] M Aicheler et.al. (Ed.), The Compact Linear Collider (CLIC) Project Implementation Plan, CERN2018-010, CERN, Geneva, 2018.

[9] M Mangano et.al. (Ed.), Future Circular Collider Study. Volume 1: Physics Opportunities, Conceptual Design Report, CERN-ACC-2018-0056, Geneva, 2018, Published in Eur. Phys. J. C. 
[10] M Benedikt et.al. (Ed.), Future Circular Collider Study. Volume 2: The Lepton Collider (FCC-ee), Conceptual Design Report, CERN-ACC-2018-0057, Geneva, 2018, Published in Eur. Phys. J. ST.

[11] M Benedikt et.al. (Ed.), Future Circular Collider Study. Volume 3: The Hadron Collider (FCC-pp), Conceptual Design Report, CERN-ACC-2018-0058, Geneva, 2018, Published in Eur. Phys. J. ST.

[12] J Jaekel, M Lamont \& C Vallee, Summary Report of Physics Beyond Colliders at CERN, CERNPBC-REPORT-2018-003, arXiv:1902.00260, CERN, Geneva, 2018.

[13] A Claudia, M Calviani, B Goddard, R Jacobsson \& M Lamont, SPS Beam Dump Facility Comprehensive Design Study, CERN-PBC-REPORT-2018-00, CERN, Geneva, 2018

[14] T Akesson et.al., Dark Sector Physics with a Primary Electron Beam Facility at CERN, CERNSPSC-2018-023, CERN, Geneva, 2018 